\title{
Development of a novel real-time PCR assay for rapid detection of African swine fever virus (ASFV) strains circulating in Vietnam
}

\author{
Thi Bich Ngoc Trinh ${ }^{1}$, Thang Truong ${ }^{2}$, Tam Nguyen ${ }^{1}$, Xuan Dang Vu ${ }^{1}$, Anh Dao ${ }^{1}$, Thi Lan \\ Nguyen $^{1}$, Aruna Ambagala ${ }^{3}$, Shawn Babiuk ${ }^{4}$, Jinsik $\mathrm{Oh}^{5}$, Daesub Song ${ }^{6}$, and Van Phan Le ${ }^{7}$ \\ ${ }^{1}$ Vietnam National University of Agriculture \\ ${ }^{2}$ Public Health Agency of Canada Ontario Manitoba Saskatchewan Regional Office \\ ${ }^{3}$ Canadian Food Inspection Agency \\ ${ }^{4}$ Affiliation not available \\ ${ }^{5}$ Median Diagnostics \\ ${ }^{6}$ Korea University \\ ${ }^{7}$ College of Veterinary Medicine. Vietnam National University of Agriculture, Vietnam
}

August 28, 2020

\begin{abstract}
African swine fever (ASF) continues to cause outbreaks throughout regions of Africa, Europe and Asia. The disease can cause severe morbidity and mortality resulting in serious economic losses. Since there is no vaccine available to control ASF, early detection is critical to contain and control the disease. The aim of this study was to develop a novel real-time PCR assay based on highly conserved ASFV gene E183L (p54). The limit of detection of the assay, VNUA-54 real-time PCR, was 2.63 copies/reaction and $2 \log 10 \mathrm{HAD} 50 / \mathrm{ml}$. The VNUA-54 real-time PCR was able to detect fifteen different ASFV reference strains representing p72 genotypes I, II and V. The assay was specific and did not amplify other swine viruses including CSF, FMD, PRRS, and PED. The diagnostic sensitivity of the real-time PCR assay was evaluated using 187 field clinical specimens collected from swine farms located in different provinces in Vietnam. The VNUA-54 real-time PCR assay is an additional tool for ASF diagnostics and can be used in combination with other p72 based ASFV real-time PCR assays as a rapid confirmatory assay.
\end{abstract}

KEYWORDS: African swine fever; real-time PCR; VNUA-P54, Vietnam INTRODUCTION

African swine fever (ASF) was first described in 1921 in Kenya (Montgomery, 1921). It is a highly lethal swine disease caused by a large double-stranded DNA virus belonging to the Asfarviridaefamily (Dixon et al., 2005). African swine fever virus (ASFV) infects exclusively suids; including domestic swine, wild boars and warthogs. The disease can cause high mortality rates up to $100 \%$ resulting in large economic losses to the swine industry in affected countries due to loss of production and trade restrictions (Costard et al., 2013). ASF transmission can occur between a healthy and an infected pig through direct contact (Costard et al., 2013) or by a bite of an ASFV infected Ornithodoros soft tick (Plowright, 1977) that is responsible for maintenance of the sylvatic cycle in Africa.

Since its first discovery in Kenya, ASF has spread outside Africa twice. The first ASF outbreak in Europe was reported in Portugal in 1957 which later spread to Caribbean and South America between the 1970s and 1980s. This outbreak was completely eradicated from Europe and Americas at a great cost with the exception of Sardinia (Sánchez-Vizcaíno et al., 2015). In 2007, ASF entered Europe for the second time 
through Georgia (Costard et al., 2013; Rowlands et al., 2008) from where it spread to the Caucasus region and Russia (Gogin et al., 2013; Khomenko et al., 2013). Then it continued its spread into Eastern Europe and entered China in 2018 where the virus become endemic (Zhao et al., 2019). Subsequently it spread to Mongolia (Heilmann et al., 2020), Vietnam (Le et al., 2019), Cambodia, Republic of Korea (H. J. Kim et al., 2020), Laos, Philippines, Myanmar and Timor-Lester, Papua New Guinea and most recently to India (OIE). Since there is no vaccine for ASF, control of this disease relies on rapid detection and elimination of the infected animals (Oura et al., 2013). Real-time PCR is the preferred first-line diagnostic for ASF. It is highly sensitive and specific, rapid and highly scalable (Fernández-Pinero et al., 2013; King et al., 2003). Several real-time PCR assays have been developed and validated for ASF detection and most of them target ASF p72 gene (Fernandez-Pinero et al., 2013; Tignon et al., 2011; A. Wang et al., 2020; Y. Wang et al., 2020; Zsak et al., 2005). Here we describe development of a novel real-time PCR assay (VNUA-P54) targeting a highly conserved region of the ASFV E183L gene that encodes an essential structural protein p54 (Brookes et al., 1998; Rodriguez et al., 1996).

\section{MTERIALS AND METHODS}

\section{Clinical samples}

One hundred and eighty-seven samples including whole blood $(n=122)$, serum $(n=12)$, spleen $(n=37)$, and kidney $(n=16)$ were collected from pigs displaying clinical signs of ASF from farms located in the different provinces in Vietnam during 2019-2020 outbreaks (Supplementary Table S4). A sample volume of $200 \mu$ l of whole blood, serum or $10 \%$ tissue homogenate were used for nucleic acid extraction using DNeasy Blood \& Tissue Kit (QIAGEN, Germany) according to the manufacturer's instructions.

\section{Determination of analytical sensitivity and specificity}

The limit of detection is defined as the highest dilution factor where $95 \%$ of the positive samples can be detected. To test the limit of detection of the VNUA-54 real-time PCR assay, 10-fold dilutions of $10^{7}$, $10^{6}, 10^{5}, 10^{4}, 10^{3}, 10^{2}$, and $10 \mathrm{HAD}_{50} / \mathrm{ml}$ of $\mathrm{VNUA} / \mathrm{HY} / \mathrm{ASF}-1 /$ Vietnam/2019 ASFV strain (Le et al.,

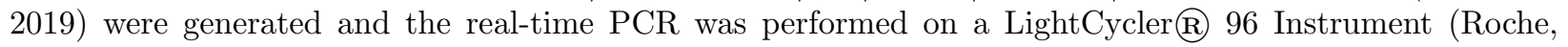
Switzerland). Additionally, the detection limit of the real-time PCR was also conducted using the known amount of DNA templates of $0.1,1,2,5$ and 10 copies for each reaction. Each DNA concentration was run 12 times by VNUA-54 real-time PCR assay as in the previous recommendation (Uhlig et al., 2015). To determine the diagnostic specificity of the real-time PCR assays, fifteen different ASFV reference strains representing p72 genotypes I, II and V (Supplementary Table S1) and other swine viruses including classical swine fever (Strain Vietnam/ND20/2014, GenBank accession no. MH979232), porcine reproductive and respiratory syndrome (Strain HUA/HP1963; GenBank accession no. KF699844), porcine epidemic diarrhea (Strain HUA14PED96, GenBank accession no. KT941120), and foot and mouth disease (Strain O/VN/PT555/2018, GenBank accession no. MN379784) were used in this study.

\subsection{Development and optimization of the VNUA-54 real-time PCR assay}

In order to design an ASFV p54 -based real-time PCR, the E183L (p54) gene sequences representing all 24 ASF p72 genotypes of ASFV were aligned by the Geneious software, and a highly conserved 100 bp region between nucleotide positions 287 and 386 was selected, and primers (Forward: 5'CAAGTGTAGGCAAGCCAGTC-3' and Reverse: 5'-GCCATGACTAGTCTGTCCGT-3') and a TaqManß) probe (5'-FAM ACGGGCAGACCGGCAACAAA-3'TAM) were designed. The primer and probe concentrations and cycling conditions were extensively optimized and the optimized reaction mixture contained $5 \mu \mathrm{L}$ of 4X TaqMan Fast Virus 1- Step Master Mix (Applied Biosystems); 10 $\mu \mathrm{M}$ of forward and reverse primers, and $10 \mu \mathrm{M}$ of probe; $5 \mu \mathrm{L}$ of extracted DNA and DNase \& RNase-free water in a $20 \mu \mathrm{L}$ reaction. The optimal thermal profile for the VNUA-54 real-time PCR assay is $50^{\circ} \mathrm{C}$ for 5 minutes; $95^{\circ} \mathrm{C}$ for 20 seconds; followed by 40 cycles of amplification $\left(3\right.$ seconds at $95^{\circ} \mathrm{C}$ and 30 seconds at $58^{\circ} \mathrm{C}$ ). All real-time PCR reactions were performed on a LightCycle ${ }^{\mathrm{TM}} 96$ (Rocher, Switzerland) and data was analyzed by the manufacturer's software. For comparison, an ASF real-time PCR developed by Tignon et al., was used as described (Tignon et al., 2011). 


\section{RESULTS}

\subsection{Analytical sensitivity of the VNUA-P54 real-time PCR assay}

The limit of detection of the VNUA-P54 assay was analyzed and compared with the validated p72-based Tignon real-time PCR assay (Tignon et al., 2011) using ASFV DNA ranging from 0.1 to 10 genome copies. Both assays were able to detect 10 and 5 ASF genome copies, while the VNUA-P54 assay detected 10/12 times and the Tignon assay 9/12 times 1 and 2 genome copies. Both assays failed to detect 0.1 ASFV genome copies (Supplementary Table S2 and 3). Based on these findings LOD was calculated for both assays using https://quodata.de/content/validation-qualitative-pcr-methods-single-laboratory. The LOD was 2.63 copies for the VNUA-P54 real-time PCR assay and 3.29 copies for the Tignon real-time PCR assay (Table 1).

The sensitivity of the VNUA-P54 assay compared with Tignon real-time PCR assay was further evaluated using a ten-fold dilution series of the ASFV strain VNUA/HY/ASF-1/Vietnam/2019. The results showed that both assays were able to detect ASFV at viral titers of $10^{7}, 10^{6}, 10^{5}, 10^{4}$, and $10^{3} \mathrm{HAD}_{50} / \mathrm{ml}$. The Ct values obtained for VNUA P54 assay were 20.78, 24.23, 27.69, 30.92, and 34.39 and Tignon assay were 22.03, $25.29,28.58,32.2$, and 35.16, respectively. In addition, at viral titer $10^{2} \mathrm{HAD}_{50} / \mathrm{ml}$, VNUA P54 assay could result in $\mathrm{Ct}$ value of 37.66 while Tignon assay was negative. Both assays failed to detect ASFV at viral titer of $10 \mathrm{HAD}_{50} / \mathrm{ml}$. Basing on the $\mathrm{Ct}$ values generated from each known virus titers, a linear relationship was observed between VNUA-P54 and Tignon real-time PCR assays (Figure 1).

\subsection{Analytical specificity of the VNUA-P54 real-time PCR assay}

To determine the analytical specificity of the VNUA-P54 real-time PCR assay, a total of 15 different ASFV reference strains of p72 genotypes I, II and V were used and the result showed that the VNUA-P54 real-time PCR assay was able to detect all of them. The specificity of the VNUA-P54 real-time PCR was also tested with other swine virus strains of CSF, FMD, PRRS, and PED and the assay did not detect any of those pathogens (Data not shown).

\subsection{Field sample evaluation of the VNUA-P54 real-time PCR assay}

A total of 187 field clinical samples including whole blood $(n=122)$, serum $(n=12)$, spleen $(n=37)$ and kidney $(\mathrm{n}=16)$ collected from pigs displaying ASF- clinical signs were tested by both VNUA-P54 and Tignon realtime PCR assays. The results showed that both assays were able to detect ASFV in all sample types. The mean Ct value of VNUA-P54 assay was 19.70 and Tignon assay was 20.83. Diagnosis results according to the type of samples showed that the mean Ct values obtained from spleen, kidney, serum, and whole blood samples were 19.01, 19.78, 24.42, and 19.44 for VNUA-P54 assay and 20.25, 20.91, 24.65, and 20.63 for Tignon assay, respectively (Table 2).

\section{DISCUSSION}

It has been almost a hundred years since ASF was first described in Kenya and today ASF is a greater threat to pig populations throughout the world. Despite numerous efforts by research groups across the globe, there is no vaccine for ASF and the disease is difficult to eradicate once established and has increased the geographic prevalence. Control of this disease relies on detection and containment and therefore rapid and sensitive diagnostics for ASF are really critical and important. Currently, there are numerous molecular and serological methods available to identify ASFV-infected animals (Abad et al., 1998; Fernández-Pinero et al., 2013; King et al., 2003; Tignon et al., 2011). Serological assays are used to determine if an animal has been exposed to ASFV. Molecular tests can detect the presence of ASFV in pigs even before to the clinical signs appear. A number of molecular assays including laboratory based, portable conventional and real-time loop-mediated isothermal amplification (LAMP) assays have been developed and validated for ASFV genome detection. Real-time PCR is the preferred assay used in many diagnostic laboratories since it is quantitative and faster compared to conventional PCR (Daigle et al.; King et al., 2003; Tignon et al., 2011; A. Wang et al., 2020; Y. Wang et al., 2020). Most of these assays are based on B646L (p72) gene. In this study, we have developed a new assay that targets ASF p54 gene. The results obtained in this study also showed that the VNUA-P54 assay is highly specific and sensitive and performs comparable to the widely used Tignon assay. 
The VNUA-P54 assay can be used to detect ASFV in different sample types including blood, serum, spleen, and kidney on its own or as an ancillary tool. The VNUA-P54 real-time PCR assay developed in this study will be an additional tool in our effort for rapid detection of ASFV in order to control the ongoing global epidemic.

\section{ETHICAL APPROVAL}

Ethical Statement is not applicable because sample collection from animals has been gathered.

\section{CONFLICT OF INTEREST}

The authors declare that there are no conflicts of interest.

\section{DATA AVAILABILITY STATEMENT}

The data that support the findings of this study are available from the corresponding author upon reasonable request.

\section{ORCID}

Van Phan Le

\section{REFERENCES}

Abad, J., Pariente, F., Hernandez, L., \& Lorenzo, E. (1998). A quartz crystal microbalance assay for detection of antibodies against the recombinant African swine fever virus attachment protein p12 in swine serum. Analytica Chimica Acta, 368 (3), 183-189.

Brookes, S., Sun, H., Dixon, L., \& Parkhouse, R. (1998). Characterization of African swine fever virion proteins j5R and j13L: immuno-localization in virus particles and assembly sites. Journal of General Virology, 79 (5), 1179-1188.

Costard, S., Mur, L., Lubroth, J., Sanchez-Vizcaino, J., \& Pfeiffer, D. U. (2013). Epidemiology of African swine fever virus. Virus research, 173 (1), 191-197.

Daigle, J., Onyilagha, C., Truong, T., Van Phan, L., Nga, B. T. T., Nguyen, T. L., . . . Ambagala, A. (2020). Rapid and highly sensitive portable detection of African swine fever virus. Transboundary and Emerging Diseases .

Dixon, L. K., Escribano, J., Martins, C., Rock, D. L., Salas, M., \& Wilkinson, P. J. (2005). Asfarviridae. virus taxonomy, eighth report of the ICTV , 135-143.

Fernandez-Pinero, J., Gallardo, C., Elizalde, M., Robles, A., Gomez, C., Bishop, R., . . . Pelayo, V. (2013). Molecular diagnosis of African swine fever by a new real-time PCR using universal probe library. Transboundary and Emerging Diseases, 60 (1), 48-58.

Gogin, A., Gerasimov, V., Malogolovkin, A., \& Kolbasov, D. (2013). African swine fever in the North Caucasus region and the Russian Federation in years 2007-2012. Virus research, 173 (1), 198-203.

Heilmann, M., Lkhagvasuren, A., Adyasuren, T., Khishgee, B., Bold, B., Ankhanbaatar, U., . . . Dietze, K. (2020). African Swine Fever in Mongolia: Course of the Epidemic and Applied Control Measures. Veterinary Sciences, 7 (1), 24.

Khomenko, S., Beltran-Alcrudo, D., Rozstalnyy, A., Gogin, A., Kolbasov, D., Pinto, J., . . . Martin, V. (2013). African swine fever in the Russian Federation: risk factors. Empres Watch, 28 , 1-14.

Kim, H. J., Cho, K. H., Lee, S. K., Kim, D. Y., Nah, J. J., Kim, H. J., . . . Choi, J. G. (2020). Outbreak of African swine fever in South Korea, 2019. Transboundary and emerging diseases, 67 (2), 473-475. 
King, D. P., Reid, S. M., Hutchings, G. H., Grierson, S. S., Wilkinson, P. J., Dixon, L. K., . . . Drew, T. W. (2003). Development of a TaqMan(r) PCR assay with internal amplification control for the detection of African swine fever virus. Journal of virological methods, 107 (1), 53-61.

Le, V. P., Jeong, D. G., Yoon, S.-W., Kwon, H.-M., Trinh, T. B. N., Lan, N. T., . . . Tuyen, N. V. (2019). Outbreak of African swine fever, Vietnam, 2019. Emerging Infectious Diseases, 25 (7), 1433.

Montgomery, R. E. (1921). On a form of swine fever occurring in British East Africa (Kenya Colony). Journal of comparative pathology and therapeutics, 34 , 159-191.

Oura, C., Edwards, L., \& Batten, C. (2013). Virological diagnosis of African swine fever-comparative study of available tests. Virus research, 173 (1), 150-158.

Plowright, W. (1977). Vector transmission of African swine fever virus. Paper presented at the Hog cholera/classical swine fever and African swine fever, Hannover (Germany, FR), 6 Sep 1976.

Rodriguez, F., Ley, V., Gomez-Puertas, P., Garcia, R., Rodriguez, J., \& Escribano, J. (1996). The structural protein p54 is essential for African swine fever virus viability. Virus research, 40 (2), 161-167.

Rowlands, R. J., Michaud, V., Heath, L., Hutchings, G., Oura, C., Vosloo, W., . . . Dixon, L. K. (2008). African swine fever virus isolate, Georgia, 2007. Emerging infectious diseases, 14 (12), 1870.

Sanchez-Vizcaino, J., Mur, L., Gomez-Villamandos, J., \& Carrasco, L. (2015). An update on the epidemiology and pathology of African swine fever. Journal of Comparative Pathology, 152 (1), 9-21.

Tignon, M., Gallardo, C., Iscaro, C., Hutet, E., Van der Stede, Y., Kolbasov, D., . . . Arias, M. (2011). Development and inter-laboratory validation study of an improved new real-time PCR assay with internal control for detection and laboratory diagnosis of African swine fever virus. Journal of virological methods, 178 (1-2), 161-170.

Uhlig, S., Frost, K., Colson, B., Simon, K., Made, D., Reiting, R., . . . Grohmann, L. (2015). Validation of qualitative PCR methods on the basis of mathematical-statistical modelling of the probability of detection. Accreditation and Quality Assurance, 20 (2), 75-83.

Wang, A., Jia, R., Liu, Y., Zhou, J., Qi, Y., Chen, Y., . . . Zhang, J. (2020). Development of a novel quantitative real-time PCR assay with lyophilized powder reagent to detect African swine fever virus in blood samples of domestic pigs in China. Transboundary and Emerging Diseases, 67 (1), 284-297.

Wang, Y., Xu, L., Noll, L., Stoy, C., Porter, E., Fu, J., . . . Dodd, K. A. (2020). Development of a real-time PCR assay for detection of African swine fever virus with an endogenous internal control. Transboundary and Emerging Diseases .

Zhao, D., Liu, R., Zhang, X., Li, F., Wang, J., Zhang, J., . . . Wu, X. (2019). Replication and virulence in pigs of the first African swine fever virus isolated in China. Emerging microbes 83 infections, 8 (1), 438-447.

Zsak, L., Borca, M., Risatti, G., Zsak, A., French, R., Lu, Z., . . . Nelson, W. (2005). Preclinical diagnosis of African swine fever in contact-exposed swine by a real-time PCR assay. Journal of clinical microbiology, $43(1), 112-119$.

\section{Table legends}

Table 1. $\mathrm{LOD}_{95}$ value of real-time PCR in detecting ASFV p54 gene with VNUA-P54 primers and P72 gene with Tignon primers.

Table 2. Diagnostic results of real-time PCR for detection of ASFV in field samples collected from different provinces in Vietnam.

Table S1 . ASFV reference strains representing for p72 genotype I, II, and V used in the study. 
Table S2. Ct values of real-time PCR in detecting p54 gene of ASFV with VNUA P54 primers and probe set.

Table S3. Ct values of real-time PCR in detecting p72 gene of ASFV with Tignon primers and probe set.

Table S4. Detail diagnostic results of real-time PCR for detection of ASFV in field samples collected from different provinces in Vietnam.

\section{Figure legends}

Fig. 1. Comparison of the detection limit between the VNUA-P54 and Tignon assays using VNUA/HY/ASF-1 /Vietnam/2019 ASFV strain.

\section{Hosted file}

Tables.docx available at https://authorea.com/users/354317/articles/477913-development-of-anovel-real-time-pcr-assay-for-rapid-detection-of-african-swine-fever-virus-asfv-strainscirculating-in-vietnam

\section{Hosted file}

Figure 1.docx available at https://authorea.com/users/354317/articles/477913-developmentof-a-novel-real-time-pcr-assay-for-rapid-detection-of-african-swine-fever-virus-asfvstrains-circulating-in-vietnam 